\title{
Phenomenon of painless knee in recurrent patellar dislocation in children
}

\author{
Adrian Masłoń ${ }^{1}$, Dariusz Witoński², Tadeusz Modrzewski ${ }^{3}$, Mateusz Grabicki ${ }^{4}$, Marcin Sibiński ${ }^{1}$, \\ Andrzej Grzegorzewski ${ }^{1}$
}

\author{
${ }^{1}$ Clinic of Orthopaedics and Paediatric Orthopaedics, Medical University of Lodz, \\ Poland \\ ${ }^{2}$ Department of Reconstructive Surgery and Arthroscopy of the Knee, Medical \\ University of Lodz, Poland \\ ${ }^{3}$ Pathomorphology and Clinical Cytopathology Department, Medical University \\ of Lodz, Poland \\ ${ }^{4}$ Orthopaedic Department, Kopernik Memorial Regional Specialized Hospital, Lodz, \\ Poland
}

Submitted: 1 December 2011

Accepted: 11 May 2012

Arch Med Sci 2014; 10, 3: 531-536

DOI: 10.5114 /aoms.2014.43747

Copyright (c) 2014 Termedia \& Banach

\begin{abstract}
Introduction: Cartilage lesion with patellar malalignment may be correlated with pain. Situations in which patients with recurrent patellar dislocation are painless between episodes of luxation can be approached with considerable curiosity. We evaluated the distribution of substance-P and S-100 protein expression in soft tissue of the knee in children with recurrent patellar dislocation, in order to evaluate the distribution of nociceptors and determine tissue origins of this situation.

Material and methods: Samples were collected from the medial and lateral synovial membrane, medial and lateral patellar retinaculum, Hoffa's body, patellar ligament, and quadriceps' aponeurosis in 10 children during the Blauth procedure and 10 adults with idiopathic osteoarthritis during total joint alloplasty. The density of nociceptive fibres was compared in the children and adults using S-100 and substance-P monoclonal antibodies.

Results: Statistical differences between groups were demonstrated for S-100 expression in synovial membrane of the medial knee compartment $(p<0.05)$ and for substance-P expression in the medial patellar retinaculum $(p<0.05)$ and synovial membrane of the lateral $(p<0.05)$ and medial $(p<0.05)$ knee compartment in favour of children.

Conclusions: Lack of pain sensations in patients with recurrent patellar dislocation may be associated with non-increased expression of nerve endings in Hoffa's body. Increased expression of either S-100 protein or substance-P in synovial membrane and the medial retinaculum did not induce pain development in the knee joints of that group of patients.
\end{abstract}

Key words: recurrence patellar dislocation, substance-P, S-100 protein, painless knee joint, children.

\section{Introduction}

Normal articular cartilage is aneural, and defects in the cartilage surface are not supposed to generate pain. However, cartilage lesion, in combination with patellar malalignment, seen in kinematic magnetic resonance images (MRI) or arthroscopy, may be correlated with pain. Ab-
Corresponding author: Adrian Masłoń MD, PhD Clinic of Orthopaedics and Paediatric Orthopaedics Medical University of Lodz 75 Drewnowska St 91-002 Lodz, Poland Phone: +48 508248265 E-mail: adian@gazeta.pl 
normal patellofemoral tracking has been reported as an important component in the development of patellar chondropathy. The term 'chondromalacia' has been used in clinical practice to denote the anterior knee pain syndrome in adolescents, that is a gross, morphological, age-related, articular cartilage softening. However, these changes may also occur without perceptible symptoms, and certain painful knees may have an apparently normal articular surface. Situations in which patients with recurrent dislocation of the patella are free of pain between episodes of luxation may be approached with considerable curiosity. Some authors have sought the origin of pain in soft tissue structures around the knee [1-4]. Numerous reports from previous studies have provided a qualitative and quantitative analysis of mechanoreceptors in knees with the anterior knee pain syndrome. However, our knowledge about the distribution of nociceptors in knees with recurrent patellar dislocation is still rather scarce [4-9]. The presence of represented free nerve endings, associated with substance $P(\mathrm{SP})$ expression in tissues of anterior knee pain syndrome knees, was described by Witoński et al. [5]. Also, previous studies have revealed that the distribution of substance $P$ nerve endings in the soft tissues around the osteoarthritic human knee or with ruptured anterior cruciate ligament $(\mathrm{ACL})$ and meniscal lesion was not age or gender dependent. S-100 protein commonly occurs in the central nervous system, astroglia and in Schwann cells, being a good marker of nervous structures in soft tissues of the knee joint, and was chosen for evaluation of nociceptors [10-12].

Both prior findings and the above-mentioned observation prompted the reported study to be undertaken, the objective of which was to evaluate the distribution of SP and S-100 peptide immunoreactivity, to evaluate the distribution of nociceptors in joints with recurrent patellar dislocation in young patients.

\section{Material and methods}

Tissue samples were collected for microscope and immunohistochemical studies from 20 patients (10 adults and 10 adolescents), treated at our centre during the years 2006-2008. Recurrent lateral patellar dislocation was the inclusion criterion for the adolescent group. The first incident of lateral patellar dislocation was evidently traumatic in character. Other patellar dislocations, in the number of 4 to 6 , resulted from small traumatic incidents. All patellar dislocations were until the operation prophylactically treated in a stabilising orthosis, combined with ice compresses, joint relief and analgesics, approved for administration in that age group, as well as exercises. The last incident of patellar dislocation took place 6 month before sur- gery. Pain sensations in the study group occurred during patellar dislocation and during a further 2-4 weeks. No pain ailments were reported in that age group, either among particular patellar dislocation episodes or before the operation. Radiological examinations of the knee joint, performed immediately after dislocation and before surgery, excluded congenital knee joint malformations. Clinical examination indicated failure of the medial retinaculum with a lateral pathway of patellar movement. The patellar compression test was positive in 3 and negative in 7 children. Intraoperative examination, done during open reconstruction of medial patellar stabilisers by the Blauth procedure, did not reveal any defects in the knee joint [13]. The mean age of the children ( 8 girls and 2 boys) at surgery was 14.3 years (age range: 10-17 years). The adult group consisted of 10 patients ( 7 female and 3 male). The mean age of the adult subjects at surgery was 66.8 years (age range: $52-83$ years); all of them were treated for osteoarthritis of the knee of idiopathic origin. Cemented total knee arthroplasty with Genesis II of Smith \& Nephew was applied in all the adult patients. None of them had previously undergone operations in the knee joint region or had been administered intra-articular steroid injections. Patients in that group reported severe pain sensations in the medial compartment of the knee joint, either constraining or totally limiting their mobility, while they did not mention any pain ailments in the patellofemoral joint. X-ray examination of the knee joint revealed degenerative changes of grade 3 and 4, following Kellgren-Lawrence's Grading Scale, that were concentrated mainly at the medial side of the knee joint [14]. The patellar compression test was positive in 5 of the patients. None of the patients required patellar resurfacing during total knee alloplasty and none reported any pain sensations of the patellofemoral joint in further follow-up.

Tissue samples for evaluation were intraoperatively collected from the following anatomical structures: medial and lateral synovial membrane (MSM and LSM), medial and lateral patellar retinaculum (MR and LR), Hoffa's body $(H)$, patellar ligament $(\mathrm{PL})$ and from the aponeurosis of the quadriceps muscle of the thigh $(\mathrm{Q})$.

\section{Material processing}

Tissue specimens, fixed in $4 \%$ formalin, were passed through a number of reagents in a Tissue-Tek VIP tissue processor of Sakura for dehydration, fluoroscopic examination and paraffin saturation.

Such processed specimens were embedded into paraffin blocks by a histoembedder of Leica and cut on a sliding microtome of Micron into $4 \mu \mathrm{m}$ sections, which were then placed on si- 
lanised microscope slides, staining the slices with haematoxylin and eosin $(\mathrm{H}+\mathrm{E})$ in an automatic Autocolor I stainer of Micron to be completed by immunohistochemical reactions: substance $P$ and $\mathrm{S}-100 . \mathrm{H}+\mathrm{E}$ staining was performed in a typical way [15].

The preparations were closed with cover glasses, using the DPX Mounting Media \& Section Adhesive media.

\section{Immunohistochemical staining}

An immunohistochemical study was performed, using the rabbit anti-substance $P$ polyclonal antibody (Chemicon International, Inc., Temecula, CA, USA) and the S-100 protein mouse monoclonal antibody (Novocastra, Newcastle upon Tyne, UK). Paraffin sections were mounted onto superfrozen slides and deparaffinised. After rehydration for $5 \mathrm{~min}$, the sections were reacted with 3\% hydrogen peroxide in distilled water and rinsed in Tris-buffered saline. Afterwards, the slides were incubated overnight in a moist chamber with the rabbit anti-substance $P$ antibody in $1: 2000$ dilution at $4^{\circ} \mathrm{C}$. The sections were then rinsed in Tris-buffered saline and a DAKO LSAB+/HRP Universal kit (DAKO A/S, Glostrup, Denmark) was used, according to the manufacturer's instructions. Positive immunoreactivity was visualised with diaminobenzidine as the chromogen. The sections were washed in distilled water, counterstained with $\mathrm{H}+\mathrm{E}$, and coverslipped with DPX mounting medium (TCS Biosciences Ltd, Buckingham, UK). Negative controls were carried out by incubation without the antibodies - the results were always negative. A similar procedure was performed with the S-100 protein mouse monoclonal antibody (Novocastra; the slides were incubated with S-100 in $1: 25$ dilution for $60 \mathrm{~min}$ ).

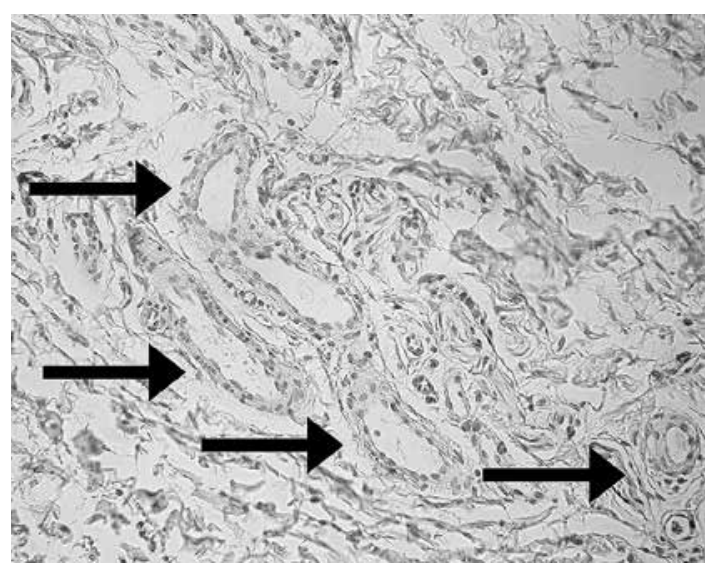

Figure 1. Synovial membrane from the lateral compartment of the knee, $\mathrm{H}+\mathrm{E}$ staining, magnification $100 \times$. Specimens with a large number of vessels (indicated by arrows)

\section{Microscope evaluation of specimens}

The specimens were examined with an Olympus BX 50 light microscope (Olympus Corporation, Tokyo, Japan).

In the $\mathrm{H}+$ E-stained specimens, the number of blood vessels was evaluated in 10 visual fields. The specimens without visible blood vessels were labelled as 0 , the specimens with a small number of vessels as 1 , those with an average number of vessels as 2 and, finally, the specimens with a large number of vessels were labelled as 3 (Figure 1).

The slices were semiquantitatively examined for substance $P$ expressing nerve fibres, which were then counted in 10 random choice visual fields (lens magnification 40x) at the centre of the microscopic field. The number of immunoreactive fibres was graded 0 (lack of nerve fibres with substance $P$ expression) or 1 (one or more immunopositive nerve fibres with substance $P$ expression). In each case, the arithmetic mean of substance P-immunopositive fibres in 10 visual fields was calculated. A similar procedure was performed for S-100 protein (Figure 2).

\section{Statistical analysis}

Statistical analyses were performed by means of SPSS 14.0 for Windows (SPSS Inc., Chicago Illinois). The Mann-Whitney $U$ test for independent samples was used to compare the number of blood vessels and the distribution of immunopositive nerve fibres, demonstrating expression for S-100 and substance $P$ in preparations collected from particular parts of the knee joint in children and adults. It was followed by a comparison of the studied groups of patients. Wilcoxon test for dependent samples was used to compare the number of blood vessels and distribution of nerve fibres - the expression of S-100 protein and sub-

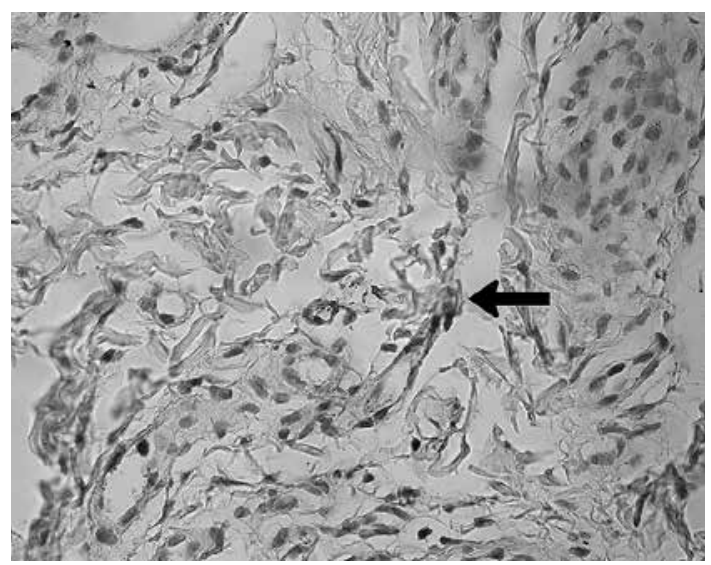

Figure 2. Synovial membrane from the medial compartment of the knee, positive S-100 immunohistochemical staining, magnification 400x. The arrow indicates the place of S-100 condensation 
stance $P$ in preparations collected from particular parts of the knee joint in children and adults. Values of $p$ below 0.05 were regarded as statistically significant.

Approval for the study was obtained from the Commission of Bioethics of the Medical University (RNN/40/06/KE of February 21, 2006).

\section{Results}

A positive immunohistochemical reaction for substance $\mathrm{P}$ and S-100 protein was obtained for all the examined tissues. In all the adolescents, a positive reaction for substance $P$ was obtained for, at least, one studied knee joint structure. A negative immunohistochemical reaction for S-100 protein in all the studied knee joint struc-

Table I. Mean value from ten consecutive visual areas showing blood vessels, substance P, S-100 protein expression in slices excised from lateral retinaculum (LR), medial retinaculum (MR), lateral synovial membrane (LSM), medial synovial membrane (MSM), fat pad $(\mathrm{H})$, quadriceps muscle tendon (Q), patellar ligament $(\mathrm{PL})$ of patients in adolescent group

\begin{tabular}{|lccc|}
\hline Variable & $\begin{array}{c}\text { Mean } \\
\text { number } \\
\text { of blood } \\
\text { vessels }\end{array}$ & $\begin{array}{c}\text { Mean value } \\
\text { S-100 }\end{array}$ & $\begin{array}{c}\text { Substance } \\
\text { P }\end{array}$ \\
\hline LR & 2.3 & 0.6 & 0.8 \\
\hline MR & 1.8 & 0.7 & 0.9 \\
\hline LSM & 2.5 & 0.8 & 1 \\
\hline MSM & 2.3 & 0.7 & 0.7 \\
\hline H & 1.7 & 0.6 & 0.6 \\
\hline Q & 1.8 & 0.6 & 0.5 \\
\hline PL & 1.5 & 0.4 & 0.5 \\
\hline
\end{tabular}

Table III. Differences in S-100 protein expression in slices excised from lateral retinaculum (LR), medial retinaculum (MR), lateral synovial membrane (LSM), medial synovial membrane (MSM), fat pad $(\mathrm{H})$, quadriceps muscle tendon $(\mathrm{Q})$, patellar ligament $(\mathrm{PL})$ of patients in groups 1-2

\begin{tabular}{|lc|}
\hline Variable & Value of $p$ \\
\hline LR & 0.063 \\
\hline MR & 0.063 \\
\hline LSM & 0.063 \\
\hline MSM & $0.023^{*}$ \\
\hline H & 0.063 \\
\hline Q & 0.280 \\
\hline PL & 0.481 \\
\hline
\end{tabular}

*Values statistically significant. Higher expression in recurrent patellar dislocation group tures was obtained in one child (Tables I and II). No statistically significant differences were demonstrated for the number of blood vessels in the specimens, collected from particular anatomical structures of adolescent and adult patients $(p>0.05)$. Statistically significant differences were demonstrated in the number of immunopositive nerve fibres with expression for S-100 protein, regarding synovial membrane of the medial knee compartment in favour of the adolescent patients $(p<0.05)$ (Table III). There were statistically significantly more immunopositive nerve fibres with expression for substance $P$ in children than in the adult patients, regarding the medial patellar retinaculum $(p<0.05)$, and synovial membrane of the lateral $(p<0.05)$ and medial $(p<0.05)$ knee compartment (Table IV).

Table II. Mean value from ten consecutive visual areas showing blood vessels, substance P, S-100 protein expression in slices excised from lateral retinaculum (LR), medial retinaculum (MR), lateral synovial membrane (LSM), medial synovial membrane (MSM), fat pad $(\mathrm{H})$, quadriceps muscle tendon $(\mathrm{Q})$, patellar ligament $(\mathrm{PL})$ of patients in adult group

\begin{tabular}{|lccc|}
\hline Structure & $\begin{array}{c}\text { Mean } \\
\text { number } \\
\text { of blood } \\
\text { vessels }\end{array}$ & $\begin{array}{c}\text { Mean S-100 } \\
\text { volume }\end{array}$ & $\begin{array}{c}\text { Mean } \\
\text { substance } \\
\text { P volume }\end{array}$ \\
\hline LR & 2.3 & 0.1 & 0.3 \\
\hline MR & 2 & 0.2 & 0.1 \\
\hline LSM & 2.8 & 0.3 & 0.1 \\
\hline MSM & 2.8 & 0.1 & 0.1 \\
\hline H & 2.5 & 0.1 & 0.2 \\
\hline Q & 1.7 & 0.3 & 0.2 \\
\hline PL & 2 & 0.2 & 0.2 \\
\hline
\end{tabular}

Table IV. Differences in substance P expression in slices excised from lateral retinaculum (LR), medial retinaculum (MR), lateral synovial membrane (LSM), medial synovial membrane (MSM), fat pad $(\mathrm{H})$, quadriceps muscle tendon $(\mathrm{Q})$, patellar ligament $(\mathrm{PL})$ of patients in groups $1-2$

\begin{tabular}{|lc|}
\hline Variable & Value of $p$ \\
\hline LR & 0.063 \\
\hline MR & $0.005^{\star}$ \\
\hline LSM & $0.015^{*}$ \\
\hline MSM & $0.023^{*}$ \\
\hline H & 0.143 \\
\hline Q & 0.280 \\
\hline PL & 0.436 \\
\hline
\end{tabular}

*Values statistically significant. Higher expression in recurrent patellar dislocation group 
Using the Wilcoxon test, we performed a cross analysis of distribution density of immunopositive nerve fibres for S-100 protein and substance $P$ and of the number of blood vessels among particular knee joint structures in the adolescent patients. No statistically significant differences were found in the distribution density of immunopositive nerve fibres for substance $P(p>0.05)$. Statistically significantly higher expression for S-100 protein was found only in synovial membrane of the lateral knee compartment vs. that in the patellar ligament. There were also more blood vessels in the lateral retinaculum vs. the medial retinaculum, a higher number of vessels in synovial membrane of the lateral compartment vs. that in the medial retinaculum, Hoffa's body, aponeurosis of the quadriceps muscle and patellar ligament, and a higher number of blood vessels in synovial membrane of the medial compartment vs. the aponeurosis of the quadriceps muscle and patellar ligament.

\section{Discussion}

Regarding the lack of pain sensations in the anterior compartment of the knee joint between dislocation incidents of recurrent patellar dislocation, their causes have not been, so far, explained. The lack of pain ailments in patellar-femoral joint instability has been noted by some authors [2, 16]. Higher expression of S-100 protein in synovial membrane of the medial knee joint compartment was observed in the group of adolescent patients vs. that in the control group of subjects with knee joint pain, resulting from degenerative disease, as well as increased expression of substance $P$ in the medial patellar retinaculum and in synovial membrane of the lateral and medial knee compartment vs. the control group. Witoński et al. did not find any differences in substance $P$ distribution in knee joints of the subjects with degenerative joint disease and in those with post-traumatic joint defects [10]. Our study demonstrated increased substance $P$ expression in the adolescent patients, regarding the medial retinaculum of the patella and synovial membrane of the lateral and medial knee joint compartment, which may have been associated with primary congestion of injured structures, nerve growth factor (NGF) and vascular endothelial growth factor (VEGF) release, which - in turn - induces excessive innervation, promotes the proliferation of vessels and substance $P$ release, and attracts mast cells [17]. No differences were found in the analysed material regarding the vascularisation of knee joint structures between the children with recurrent patellar dislocation and the adult subjects from the control group with degenerative disease in the course which synovial membrane congestion was observed [18]. The lack of ischaemic changes and of pain ailments seems to confirm the theory of Sanchis-Alfonso that implicates ischaemic changes to be responsible for pain in the anterior knee joint compartment. Those authors found histological changes, resulting from hypoxia in the lateral retinaculum, which was responsible for pain in the anterior knee joint compartment [2]. That result may be likely to prompt a hypothesis that local congestion of soft tissues, being the expression of inflammatory status, was similar in the patients of both groups, thus not predisposing to tissue hypoxia.

The role of the lateral retinaculum in the pathology of pain in the anterior knee pain syndrome has been documented fairly well and in detail. Fulkerson explains it by an adaptive shortening of the lateral retinaculum, which undergoes stretching during knee bending, when the patella is dislocated towards the medial part. This mechanism may lead again to innervation changes on the lateral side of the knee [19]. Other results were presented by Witoński and Wągrowska-Danielewicz, who found a higher number of free, substance $P$ containing nerve endings in the medial retinaculum of the knee and in Hoffa's body in patients with anterior knee pain syndrome than in patients with degenerative disease of the knee joint and without these pain symptoms. That dependence was, however, observed in the lateral retinaculum in the three groups of examined patients. Earlier studies, performed by the second authors (DW), demonstrated uniform substance $P$ distribution in knees affected by degenerative disease and after knee joint injuries in adult subjects [5]. In our study, higher volumes of substance $P$ and S-100 protein were found in certain structures of the knee joint in children vs. those in adult subjects. Statistically significant differences occurred in the medial retinaculum and synovial membrane, both on the lateral and medial side, for substance $P$ and in synovial membrane on the medial side of the anterior compartment of the knee. There was, however, no increased expression of either S-100 protein or substance $P$ in Hoffa's body vs. the group of adult subjects with degenerative disease and pain sensations localised mainly beyond the patellofemoral joint. The patients reported pain ailments mainly from the medial compartment, which may indicate a decisive role of Hoffa's body in pain development of the anterior compartment of the knee. That hypothesis was confirmed by a study of Bohnsack et al. [20], who, while studying the distribution of substance $P$ and S-100 protein in Hoffa's body and in the adjacent synovial membrane, found nerves of small diameter in Hoffa's body, while nerves of medium and large diameters were present in the surrounding synovial membrane. That observation 
confirms the hypothesis about the presence of terminal nerve endings in Hoffa's body and a meaningful role of the structure in pain development in the anterior compartment knee joint.

The obtained results support the theory that children with recurrent patellar dislocation are less predisposed to pain development in the anterior compartment of the knee, despite a higher number of immunopositive nerve fibres with expression for substance $P$ in the medial knee retinaculum and synovial membrane, both on the lateral and medial side, as well as the presence of fibres with expression for S-100 protein in synovial membrane of the medial compartment with no increased expression within Hoffa's body. The results of our studies seem to confirm the dominant role of Hoffa's body as a source of pain in the anterior knee pain syndrome, which is a specific phenomenon, demanding further studies. The lack of a control group with adult subjects without any pain sensations in the knee joint, especially in its anterior compartment, may be regarded as a weak point in our study. Taking into account the ethical and legal aspects, it is impossible to obtain material for studies. Nevertheless, it seems that the group of patients with degenerative disease of the knee joint, manifested by varus bending of the limb axis and pain sensations, localised mainly in the medial part of the joint and with scarce ailments from the patellofemoral joint, may be approached as a group without pain ailments from the anterior compartment of the knee. Evidence supporting this view has been found in the lack of post-operative ailments from the patellofemoral joint without patellar resurfacing.

In conclusion, both the reported observations and previous findings indicate that the lack of pain sensations, characteristic for the anterior knee pain syndrome, in the anterior compartment of the knee of patients with recurrent patellar dislocation, may be associated with non-increased expression of nerve endings in Hoffa's body. Increased expression of either S-100 protein or substance $P$ in synovial membrane and the medial retinaculum did not induce pain sensation in the knee joints of that group of patients.

\section{References}

1. Lembeck F, Folkers K, Donnerer J. Analgesic effect of antagonists of substance P. Biochem Biophys Res Commun 1981; 103: 1318-21.

2. Sanchis-Alfonso V, Roselló-Sastre E, Monteagudo-Castro C, Esquerdo J. Quantitative analysis of nerve changes in the lateral retinaculum in patients with isolated symptomatic patellofemoral malalignment. A preliminary study. Am J Sports Med 1998; 26: 703-9.

3. Walsh DA, Salmon M, Mapp PI, et al. Microvascular substance $\mathrm{P}$ binding to normal and inflamed rat and human synovium. J Pharmacol Exp Ther 1993; 267: 951-60.
4. White DM, Helme RD. Release of substance P from peripheral nerve terminals following electrical stimulation of the sciatic nerve. Brain Res 1985; 336: 27-31.

5. Witoński D, Wagrowska-Danielewicz M. Distribution of substance-P nerve fibers in the knee joint in patients with anterior knee pain syndrome. A preliminary report. Knee Surg Sports Traumatol Arthrosc 1999; 7: 177-83.

6. Bohnsack M, Hurschler C, Demirtas T, Rühmann O, Stukenborg-Colsman C, Wirth CJ. Infrapatellar fat pad pressure and volume changes of the anterior compartment during knee motion: possible clinical consequences to the anterior knee pain syndrome. Knee Surg Sports Traumatol Arthrosc 2005; 13: 135-41.

7. Leunig M, Beck M, Stauffer E, Hertel R, Ganz R. Free nerve endings in the ligamentumcapitisfemoris. Acta Orthop Scand 2000; 71: 452-4.

8. Biedert RM, Stauffer E, Friederich NF. Occurrence of free nerve endings in the soft tissue of the knee joint. A histologic investigation. Am J Sports Med 1992; 20: 430-3.

9. Witoński D, Wagrowska-Danilewicz M, Raczyńska-Witońska G. Distribution of substance $P$ nerve fibers in osteoarthritis knee joint. Pol J Pathol 2005; 56: 203-6.

10. Zimmer DB, Cornwall EH, Landar A, Song W. The S100 protein family: history, function, and expression. Brain Res Bull 1995; 37: 417-29.

11. Fanò G, Biocca S, Fulle S, Mariggiò MA, Belia S, Calissano $P$. The S-100: a protein family in search of a function. Prog Neurobiol 1995; 46: 71-82.

12. Kashani IR, Golipoor Z, Akbari M, et al. Schwann-like cell differentiation from rat bone marrow stem cells. Arch Med Sci 2011; 7: 45-52.

13. Blauth W, Mann M. Medial-and simultaneous anterior-transfer of the tibial tuberosity. Z Orthop Ihre Grenzgeb 1977; 115: 252-5.

14. Kellgren JH, Lawrence JS. Radiological assessment of osteo-arthrosis. Ann Rheum Dis 1957; 16: 494-502.

15. Burck HC. Technika histologiczna [Polish]. PZWL, Warsaw 1975.

16. Dye SF, Staubli HU, Biedert RM, Vaupel GL. The mosaic of pathophysiology causing patellofemoral pain: therapeutic implications. Oper Tech Sports Med 1999; 7: 46-54.

17. Sanchis-Alfonso V, Roselló-Sastre E, Revert F, García A. Histologic retinacular changes associated with ischemia in painful patellofemoral malalignment. Orthopedics 2005; 28: 593-9.

18. Smith MD, Triantafillou S, Parker A, Youssef PP, Coleman MJ. Synovial membrane inflammation and cytokine production in patients with early osteoarthritis. Rheumatol 1997; 24: 365-71.

19. Fulkerson JP. The etiology of patellofemoral pain in young active patients: a prospective study. Clin Orthop Relat Res 1983; 179: 129-33.

20. Bohnsack M, Meier F, Walter GF, et al. Distribution of substance-P nerves inside the infrapatellar fat pad and the adjacent synovial tissue: a neurohistological approach to anterior knee pain syndrome. Arch Orthop Trauma Surg 2005; 125: 592-7. 